\title{
L'entretien avec Jean-Paul Sartre. Le questionnaire implicite du discours sur la littérature des écrivains Verdier
}

\author{
Mathilde Barraband \\ Université du Québec à Trois-Rivières
}

Une scène, symbolique, pourrait éclairer la place singulière qu'occupent les éditions Verdier dans le paysage intellectuel français contemporain. Cette scène, c'est celle de la rencontre, ou plutôt des rencontres, au cours des années 1970, de JeanPaul Sartre et de Benny Lévy. Le vieil intellectuel et le jeune dirigeant de la Gauche prolétarienne se rencontrent alors pour préparer la publication d'un long entretien, au titre significatif : « Pouvoir et liberté » (Sartre, 1979, p. 14). L'histoire n'a souvent retenu de cet épisode que le recentrement des deux hommes autour de la question juive, le «détournement» même du 
philosophe existentialiste et athée par Pierre Victor, redevenu Benny Lévy. Pourtant, le dialogue s'est engagé plus largement autour d'une question essentielle pour ces deux chefs : celle de l'autorité politique et intellectuelle. Et l'influence ici semble avoir été réciproque : si les réflexions de Sartre ont poussé Lévy à la dissolution de la Gauche prolétarienne, Sartre souhaitait commencer leur livre d'entretien par la « décomposition » de sa propre figure comme intellectuel engagé (Lévy, 2007, p. 9-14). Les deux chefs idéologiques, l'un ayant constamment tribune dans les médias et l'autre œuvrant à la tête d'une organisation semi-clandestine, ont ainsi entrepris de dresser, en ce début des années 1980, le bilan de l'expérience révolutionnaire des dernières décennies, tirant ensemble le rideau sur une période.

Les éditions Verdier naissent doublement de cet entretien continué entre Sartre et Lévy. Tout d'abord, parce que la maison est créée en 1979 par d'anciens militants de la Gauche prolétarienne ${ }^{1}$, qui, faisant «le point sur les années gauchistes, sur l'inabouti partiel de ces années-là » (Bobillier, 1992), ont voulu poursuivre la lutte par d'autres moyens. Ensuite, parce que ce dialogue a fourni à la jeune maison sa matière première : c'est elle qui s'est fait le principal éditeur des textes qui en sont issus ${ }^{2}$. La jeunesse des années 1970, qui en ce temps raillait Sartre comme cet intellectuel à la solde du parti communiste, fut celle-là même qui, après la mort du philosophe et jusqu'à

1 Gérard Bobillier, Colette Olive, Michèle Planel, et Benoît Rivéro, selon Bobillier (1992).

2 Tous ces textes sont issus des sept années de rencontre entre Lévy et Sartre : Le Nom de l'homme: dialogue avec Sartre (1984); L'Espoir maintenant: les entretiens de 1980 (avec Jean-Paul Sartre), suivis du Mot de la fin (1991); La Cérémonie de la naissance, (2005); Pouvoir et liberté (cahiers de travail en dialogue avec Sartre, 1975-1980) (2007). 
aujourd'hui, contribua à le maintenir dans le paysage de la pensée.

Les éditions Verdier, d'abord attachées à accompagner Lévy dans son nouveau chemin spirituel $^{3}$, se tournèrent progressivement vers la littérature contemporaine, s'y consacrant très largement dès la fin des années 1980. Au tournant du siècle, elles étaient parvenues à fédérer autour d'elles des auteurs, publiés jusqu'alors dans diverses maisons d'édition, dont il est difficile de ne pas remarquer qu'ils appartenaient à la même génération et avaient en commun un passé gauchiste. Pierre Bergounioux, François Bon, Didier Daeninckx, Pierre Michon, Olivier Rolin et Natacha Michel, notamment, qui ont rejoint Verdier entre 1988 et $2000^{4}$, sont nés, comme les fondateurs de Verdier, entre 1941 et $1953^{5}$, et ont commencé à publier pour la plupart dans la première moitié des années 19806. L'aventure Verdier semble bien, comme le formule le catalogue de la maison, avoir rassemblé "sans mot d'ordre tous les esprits et les énergies de ceux qui avaient su garder dans l'oreille la petite musique de Mai et la volonté de ne pas s'arrêter à l'échec des années de militantisme

\footnotetext{
3 La colonne vertébrale du catalogue des éditions Verdier fut d'abord constituée de textes spirituels, en majorité hébraïques. Vinrent ensuite les premiers essais de sciences humaines, puis la littérature française.

4 Michon publie pour la première fois aux éditions Verdier en 1988, Bergounioux et Bon en 1992, Daeninckx en 1993, Michel en 1998 et Rolin en 2000.

5 Michel est née en 1941, Michon est né en 1945, Rolin en 1947, Bergounioux et Daeninckx en 1949, Bon en 1953.

6 Entre 1982 et 1985 paraissent les premiers romans de François Bon chez Minuit, d'Olivier Rolin au Seuil, de Pierre Michon, de Pierre Bergounioux et de Didier Daeninckx chez Gallimard. Seule Natacha Michel a commencé a publié chez Gallimard dès 1973.
} 
politique ${ }^{7}$ ». Dès lors, on peut se demander quelle trace de cette reconversion demeure dans la conception de la littérature de ces écrivains. Si leurs œuvres trouvent des formes singulières, seraient-elles néanmoins portées par un projet similaire ou, du moins, une idée semblable de ce que peut, cherche la littérature? «Ce qui lie en arrière-fond [Bergounioux, Bon, Daeninckx, Michel, Michon et Rolin], suggère en ce sens une des éditrices Verdier, c'est quand même le souci, la question du politique - posée de manière sous-jacente ou pas ${ }^{8}$ ». Comment, dès lors, se nouent littérature et politique pour cette génération qui a voulu enterrer la littérature engagée - et, parfois, la littérature tout court —, puis a abandonné le militantisme pour l'écriture? Comment ces écrivains, rassemblés par une maison d'édition qui semble être née de la réconciliation de la génération 68 avec Sartre, gèrent-ils l'héritage de ce dernier? En somme, il s'agit d'observer comment la scène initiale de la fondation des éditions a pu essaimer dans le discours sur la littérature de ses auteurs phares.

\section{Absences de Sartre}

De toute évidence, le nom de Sartre n'est pas celui qui est le plus souvent invoqué par Bergounioux, Bon, Daeninckx, Michon, Michel ou Rolin. Il est très rarement cité dans les essais, à peine plus dans les entretiens. Le seul relevé des occurrences

\footnotetext{
7 Verdier, 30 ans d'édition, 1979-2009 (2009, p. 3-4). Le texte n'est pas signé, conformément à l'idée d'un nécessaire retrait des éditeurs. La conclusion ajoute: «Si on ne pouvait pas changer le monde on pouvait du moins contribuer à changer les consciences. »

8 Entretien personnel avec Michèle Planel, le 20 mai 2009. Nous remercions très vivement les éditeurs Verdier pour le dialogue qu'ils ont toujours accepté de nouer avec simplicité et générosité.
} 
de son nom inviterait à conclure à une éclipse de la figure sartrienne du champ référentiel de ces auteurs. À la rareté des invocations s'ajoute le fait qu'elles n'ouvrent presque jamais sur une véritable discussion autour de sa pensée ou de ses œuvres littéraires. À peine prononcé, le nom de Sartre disparaît, comme si l'on s'entendait sur ce qu'il appelle. Mais cette économie est aussi le signe que le vocable s'est fait emblème. Il n'est à cet égard pas anodin qu'il apparaisse très souvent comme élément d'une liste ou, alors, dans un jeu d'opposition. Sartre, c'est l'écrivain engagé, comme Aragon, Camus, c'est encore celui qui s'est trompé d'engagement ou dans la forme de son engagement, pas comme Blanchot, Bataille, ou Barthes. On lui oppose encore d'autres modèles, notamment celui de « créateurs », Gracq ou Simon, qui ont été de véritables artisans, quand Sartre a voulu se placer en théoricien. La figure sartrienne, certes effacée, devenue emblème, continue ainsi clairement d'organiser les représentations de la littérature, ne serait-ce que comme repoussoir.

De quoi Sartre reste-t-il alors le nom pour ces auteurs? Pas de la littérature, si l'on en juge par l'absence totale de référence à ses œuvres littéraires ou à ses techniques d'écriture. Il reste en tant que penseur de la littérature ou, plutôt, "directeur de conscience» (2007, p.15), comme dit Michon, ce censeur qui aura inventé des paradigmes qui continuent d'orienter souterrainement la réception de la littérature : «Sartre, écrit François Bon, [...] a fait beaucoup de mal avec ses lectures réductrices, parce que, excellent philosophe enfin j'espère, il ne comprenait rien à la littérature.» (Bon, 2005) Et l'écrivain d'incriminer, en particulier, son «opposition simpliste de la poésie-essence et du roman-réalisme » et son idée que le salut des prosateurs est 
dans l'engagement. Bergounioux se livre, lui aussi, à une condamnation sévère du critique et théoricien. Doctorant de Barthes, il reprochait déjà à Sartre de manier les textes " comme des documents ${ }^{9}$ »; trente ans plus tard, il accuse encore l'auteur de l'Idiot de la famille ${ }^{10}$ d'avoir été trop occupé à illustrer ses thèses existentialistes, trop empêtré dans son idéalisme, sa «liberté », pour comprendre Flaubert. En somme, pour ces écrivains contemporains, Sartre n'est ni écrivain, ni du côté des écrivains. Rejeté entièrement du côté d'une théorie fallacieuse et inhibitrice, il est encore critiqué pour ses engagements. Rolin rappelle que l'antiintellectualisme de la Gauche prolétarienne, dont il faisait partie, s'était largement nourri de l'idée que l'intelligentsia française, aussi brillante fût-elle, et Sartre en premier lieu, avait failli pendant la Seconde Guerre mondiale, ne sachant pas prendre à temps la mesure de la terreur du nazisme (2008, p. 155). Et il avoue que la défiance ne l'a pas tout à fait quitté. C'est en fait la question de l'autorité qui continue de poser problème dans le concept d'écrivain engagé. Savoir écrire, comme le formule Bergounioux, reprenant Simon, n'autorise pas à donner son avis sur des questions générales (2000, p. 154). S'il lui arrive de le faire, c'est, dit-il, «du bout des lèvres », parce qu'on l'y convie, et le moins souvent possible. Inversons les propositions, et l'on obtiendra une bonne

9 «J.-P. Sartre, dans l'étude qu'il a faite de la constitution et de la personnalisation de Flaubert dans ses phases successives [n'utilise] les textes de celui-ci qu'au titre de documents, sans égard pour les caractéristiques formelles, les signes littéraires qu'ils présentent », écrit Bergounioux (1975, p. 76). Dans L'Héritage (2002, p. 120), il n'est pas plus tendre avec l'auteur de L'Idiot de la famille, qu'il décrit "empêtré dans son existentialisme, son idéalisme, sa "liberté" ».

10 En 1971, quand le premier volume de l'Idiot de la famille paraît, Bergounioux vient de commencer sa thèse. 
définition de l'intellectuel selon le modèle sartrien : qui parle haut et fort, surtout lorsqu'on ne l'y invite pas, et le plus souvent possible. Sartre fut en effet, selon les images consacrées, le «Veilleur de nuit sur tous les fronts de l'intelligence » (Audiberti, cité par Raimond, 1989) et « celui qui s'occupe de ce qui ne le regarde pas" (Sartre, 1972, p.12). Daeninckx lui-même prend ses distances avec la figure sartrienne et avec le terme même d'« engagement » qui, selon lui, a «souffert des combats du siècle» (1999, p. 23). Il reproche à Sartre d'avoir accepté de devenir le propagandiste d'une idéologie, d'un parti. «Généreux dans ses prises de positions, dit-il, un écrivain peut se brûler à la flamme qu'il tend pour éclairer les autres. » (p. 24) Pour sa part, Daeninckx se voit plutôt comme un "franc-tireur » (p. 24), qui ne veut inscrire sa parole sous aucune autorité, inféoder son travail de dévoilement à aucune fin qui le dirige, donc le dépasse et le subordonne.

\section{Le dialogue continué}

Si le nom de Sartre est peu prononcé, ses textes rarement cités, et si sa figure et ses écrits se résument alors le plus souvent à la question, jugée obsolète, de l'engagement, le discours sur la littérature des écrivains Verdier n'est pas quitte pour autant de l'héritage sartrien. Les problématiques précises que soulève notamment Qu'est-ce que la littérature?, les distinctions que Sartre opère, les concepts qu'il forge, travaillent encore leurs questionnements. Qu'est-ce qu'écrire? Pourquoi écrit-on et pour qui? forment ainsi le questionnaire implicite de leurs réflexions, qui s'élaborent dans un dialogue contradictoire avec les réponses que Sartre lui-même y a apportées. Les discours 
sur la littérature des six écrivains Verdier, venant donner la réplique à un même texte originel, entrent dès lors en résonance.

Les positions de Bergounioux, Bon, Michel, Michon, Rolin ou Daeninckx sont loin d'être superposables et, à bien des égards, le dernier fait figure d'exception. Cependant, une objection commune est adressée par tous ces auteurs au principe d'une subordination de l'esthétique à l'éthique, d'une mise au pas de la forme par le message. Le discours de Daeninckx lui-même, s'il puise largement dans le vocabulaire sartrien, s'attache à opérer des distinctions dont la véritable motivation semble être de réserver un espace à la dimension strictement esthétique de son entreprise littéraire. Depuis Meurtre pour mémoire, il place au cœur de son projet une mission morale de révélation des données occultées de l'histoire française. " On ne peut pas continuer à vivre tant que certaines choses ne sont pas dites, ni nommées», affirme l'écrivain avec des accents sartriens. «Dévoiler de tels événements n'est pas facile, mais, pour moi, c'est de l'ordre de la prise de responsabilité, et c'est une histoire de dignité. » (p. 22) L'écriture est quelque chose de grave, et l'écrivain n'a pas à être jugé irresponsable, puisqu'il engage sa pensée et son savoir dans ses œuvres. Mais la question de la forme ne saurait cependant venir par-dessus le marché, explique Daeninckx, dont l'ambition est de réconcilier ce que l'histoire de la littérature française a disjoint dans ses représentations : c'est-àdire l'acuité d'un regard sur la société et la vivacité de la langue comme de la structure, le «réalisme » et un "modernisme » (p.10), dit-il. Le livre a en quelque sorte, chez Daeninckx, un double acte de naissance: le moment de la découverte d'un événement caché, puis celui de la trouvaille formelle. Et l'un et 
l'autre sont d'égale importance. Le producteur du livre, de même, est double : il va au combat, mais en tant que citoyen et non en tant qu'écrivain (p.24-25). Par ce jeu de masques, Daeninckx recrée, au sein même d'une démarche politique, l'espace d'une autonomie pour la littérature.

\section{Qu'est-ce qu'écrire?}

Il reste que le livre se présente, chez Daeninckx, comme un médiateur entre le savoir de celui qui l'écrit et ceux qui le recevront. "Si personne ne le dit, à quoi nous sert-il de le savoir? », questionne l'exergue de Camarades de classe, citant Qu'est-ce que la littérature? Ici réside un point de basculement qui distingue Daeninckx et Sartre d'une part, et les autres écrivains Verdier d'autre part. Pour ces derniers, le savoir n'est pas à la source de l'œuvre. Il y a plutôt une connaissance à l'œuvre dans la littérature. À Sartre, ils préfèrent alors Simon, qui a attaqué dans son Discours de Stockholm la façon dont le philosophe avait pu se constituer en détenteur, en propriétaire en quelque sorte, d'un savoir : " "Qu'avez-vous à dire?" demandait Sartre - en d'autres termes : "quel savoir possédezvous?" » (p. 14). Un des nœuds du discours sur la littérature de Bergounioux, Bon, Michel ou Rolin repose sur cette tentative de définir le rapport complexe du processus littéraire à la connaissance. Comparant la littérature et la philosophie, Michel pose par exemple ces distinctions :

Ce n'est pas que la littérature ne [dise pas la vérité], ni qu'elle soit totalement immune à sa propre époque. La grande différence est que la philosophie dit et la littérature fait. [...] ce n'est pas que la philosophie est théorique et la littérature pratique. Encore une fois, ce n'est pas cela. Ni même que l'une fait ce qu'elle doit en le sachant, et l'autre en ne le sachant pas. 
[...] C'est que [c]'est en faisant ce qu'elle fait [que la littérature] voit la vérité. (p. 12-14, Michel souligne)

Il y a, précise pour sa part Bergounioux, une différence entre le dévoilement qu'opèrent la littérature et le savoir que délivrent les sciences humaines. La littérature ne délivre pas un savoir sur l'homme ou la société. Et si elle a pu le faire, c'était par défaut, avant que les sciences sociales ne se constituent. Aussi, si le citoyen veut s'informer, est-ce vers ces dernières qu'il doit se tourner, et non vers la littérature (Bergounioux, 2001, p. 35). Enfin, rappelant l'épisode des paroles gelées chez Rabelais, Bon commente: «il ne va pas de soi que nommer ce qui ne se connaît pas n'en ramène pas forcément une parole de connaissance, et le fait proprement littéraire se joue là » (1993).

En deçà de leurs singularités, ces efforts de définition se rejoignent sur cette idée que la littérature se distingue de tous les autres discours sur le monde en ceci qu'elle ne divulgue pas un savoir positif, réutilisable, exploitable. On est loin ici du critère sartrien de la lisibilité. La littérature est pensée, explique Rolin, mais pensée métaphorique et non pas apodictique ${ }^{11}$. La pensée littéraire n'existe pas ailleurs que dans sa forme ou dans un processus de modélisation, d'incarnation ou de figuration, qui fait d'elle un art du détail, ainsi qu'il l'explique dans un de ses entretiens :

Quand je suis venu à la littérature, j'ai eu le sentiment que je dérogeais, que je faisais une chose impure, superficielle et impressionniste, que ce n'était pas de l'ordre du savoir. Maintenant, trente ans plus tard, je pense que si, que c'est un savoir et même un savoir plus profond, à beaucoup d'égards plus aigu que le savoir philosophique ou historique. Mais c'est

11 Olivier Rolin, Conférence à la Sorbonne, Séminaire de Marc Dambre, 13 février 2002. Je transcris. 
un savoir [...] qui procède par visions, par tableaux, et aussi par nuances. (2008, p. 150-151)

Une constante du discours des écrivains Verdier est alors de réaffirmer la capacité d'élucidation - quels que soient ses chemins - de la littérature. Cette réaffirmation est notamment au cœur de l'essai de Michel, qui appelle de ses vœux un écrivain pensif, qui ne soit ni l'écrivain-intellectuel dont le modèle a vécu, ni l'écrivain-artiste qui «écrit au génie [...] surtout pas au projet» (p. 22) et qui, «au nom de mille arguments: le terrorisme de la pensée, vieille antienne, son inutilité, sa désuétude [...], abandonne sa part de lumière » (p. 17). Dès lors, se pose la question du destinataire. Pour qui est cette élucidation? L'écrivain ne l'engage-t-elle que pour luimême? Le questionnaire de Sartre vient ici à nouveau tarauder le discours de ces écrivains.

\section{Pour qui écrit-on?}

Chez Bon, Bergounioux, Michel, Michon et Rolin, on trouve clairement énoncé que le texte littéraire ne tend vers aucun destinataire. Au moment de l'écriture, le lecteur est absent, affirment Bergounioux et Bon ${ }^{12}$. Rolin tance sévèrement : «un écrivain ne doit pas "s'adresser" à telle ou telle catégorie sociologique plutôt qu'à une autre, ou bien alors c'est un commerçant» (2008, p.154), et Michon souligne plus empiriquement une contradiction : "l'écrivain est double : on fait le texte en pensant peu à la réception, après la publication

\footnotetext{
12 Quelques exemples: «On ne pense pas au lecteur, lorsqu'on écrit. On combat le chevalier noir » (Bergounioux, 2002, p. 29); ou « On ne donne rien à lire. On cherche dans sa tête à soi ce qui permet de conjurer temporairement la peur, ou le désarroi, ou la culpabilité » (Bon, 1995).
} 
on est un autre qui attend les lauriers et qui n'est pas vraiment celui qui a écrit» (2007, p. 292). Ailleurs, Michon précise de quoi ressortit selon lui ce rapport distant que l'écrivain entretient, dans l'écriture, à son lecteur :

On écrit comme jadis on s'adressait [...] à une grande instance fantasmatique mais comblante, qu'on appelait Dieu. [...] Dieu n'est autre que cette assomption d'autrui dans une instance transcendante. De cette assomption, l'écrivain a besoin pour que son texte se hausse au-dessus de celui qui l'écrit. (p. 29-30)

Refus de la destination ou dédicace transcendante, ces perspectives reviennent à éliminer le lecteur — réel ou virtuel - comme instance sociale du champ de perception de l'écrivain au travail. Nous sommes loin, ici, de l'idée sartrienne d'un nécessaire alignement de l'écriture sur le destinataire. On peut choisir d'écrire certaines choses et de le faire pour le plus grand nombre, résume Bergounioux, mais "l'œuvre devra alors se mouler sur une attente socialement, scolairement conditionnée, qui accuse un retard de cinquante ans sur la littérature contemporaine ${ }^{13}$ ». "Le dilemme de la forme n'a rien de formel », conclut-il, « l'alternative est embarrassante mais on a le choix » (Bergounioux, 2001).

Ces affirmations très tranchées sont tout de même modulées ailleurs. Bergounioux dit penser de plus en plus à ce jeune lecteur auquel il serait heureux d'apporter des réponses. Rolin admet qu'une des motivations de Tigre en papier, « c'était

13 Cette tentation, explique Bergounioux, est d'autant plus forte pour les écrivains contemporains qu'ils n'appartiennent plus désormais à la seule classe minoritaire de ceux qui héritaient et de l'instruction et de la fortune. Grâce à la généralisation de l'accès à l'enseignement secondaire, de nouvelles classes sociales accèdent à la création, mais la posture désintéressée de leurs prédécesseurs leur est plus difficilement accessible. (Bergounioux, 2001, p. 35) 
cela : m'adresser à des jeunes gens, même si, encore une fois, je ne suis pas démagogue jusqu'au point de prétendre que c'était fait pour eux » (2008, p. 154). Par tant de fermeté, il s'agissait surtout, semble-t-il, de dégager la parole littéraire des structures de la communication. La littérature, sans message, ne saurait assumer une fonction informative et, sans destinataire, elle ne saurait non plus comporter une fonction conative. Il s'agissait enfin de régler son compte à l'appel sartrien pour une littérature qui soit une arme de combat. «Il n'est plus permis, explique Bergounioux, un siècle et demi après [Flaubert] de créditer l'“Art” d'une puissance opératoire qui l'élèverait au rang des déterminismes agissants. » Et de poursuivre :

Qui prétend, aujourd'hui, non pas suspendre mais simplement infléchir la marche du monde n'ira pas tracer des chimères dans le silence d'une chambre. Il fera, comme on dit, de la politique parce que c'est elle qui constitue, à cette échelle, le plan approprié d'interprétation et d'intervention [...]. Toucher, maintenant, à la littérature, cela suppose qu'on en sait les limites. (Bergounioux, 1995)

Bon va exactement dans le même sens. Lorsque Jean-Claude Lebrun l'interroge en 1998 sur son « éloignement par rapport au genre [romanesque] qui pourrait être perçu comme une prise de distance par rapport à ses fonctions sociales », il répond : «La chance que j'ai eue, c'est d'avoir compris très tôt que je n'ai pas mon mot à dire là-dedans. [...] Voilà la condition de l'écriture : si on se fait une quelconque illusion sur l'effectivité, sur la réception, sur la lisibilité, ce n'est rien. » (Bon, 1998) En somme, pour ce qui est de la description du processus littéraire, ces écrivains suivent le cheminement de Sartre jusqu'à un certain point, d'où ils bifurquent radicalement: certes, nommer, c'est faire exister, peut-être 
éclairer, dévoiler, mais dévoiler n'est pas changer, écrire n'est pas agir, ni faire agir.

Le destinataire congédié, ou porté hors d'atteinte, il ne resterait plus qu'à suspendre l'écrivain en haut de sa colonne, son stulos, dont Rolin rappelle qu'il est à l'origine du mot «style ». C'est ce que semble faire Bergounioux lorsqu'il affirme que "[l]a tâche de l'écrivain, à la fin du xxe siècle, s'est rapprochée de la définition qui est la sienne, qui n'est pas d'investir le domaine politique mais, en solitaire, souvent contre la doxa, d'interroger le monde qui l'entoure " (2001, p. 35). Écrire, affirme-t-il en écho à Henri Michaux, c'est « chercher, solitaire, à l'écart du sens commun, derrière les apparences, le grand secret.» (p.35) Ce positionnement paradoxal, l'affirmation de l'écriture contre la loi commune sont des idées force que développent avec une même constance Rolin ou Michon. L'écrivain est fondamentalement, selon eux, celui qui entretient un rapport décalé à la langue : "Le style c'est ce qui déroge à l'usage » (2008, p.156), rappelle le premier, renvoyant à Barthes ${ }^{14}$. L'écrivain est un pirate, explique le second, citant Pierre Bourdieu : il tente des coups, il essaie et, plus précisément, il essaie d'éviter les lieux communs (2007, p. 323). Or ce piratage suppose d'avoir écumé les eaux communes, d'en avoir expérimenté les écueils et même de suivre d'une certaine manière le courant de son époque : les lieux communs, en effet, se déplacent sans arrêt, et plus vite que jamais à l'ère du "grand Communicant» (p. 323). Suivant d'autres métaphores et prolongeant la piste étymologique du mot «style », Rolin souligne la même contradiction : la colonne est ce qui se dresse, ce qui fait support, mais encore ce sur quoi

\footnotetext{
14 Voir Olivier Rolin, Conférence à la Sorbonne, loc. cit.
} 
on monte pour mieux voir. L'écrivain ne peut, de ce fait, être un étranger ni à la société qui le porte ni à son temps.

Ainsi, le discours des écrivains Verdier hésite à situer l'écrivain hors du groupe ou hors de son temps, de même qu'il répugne à congédier tout à fait le destinataire. Les images employées pour traduire le positionnement de l'écrivain travaillent à définir un espace qui n'est pas un à-côté nettement séparé, mais un écart, c'est-à-dire un lieu en tension. Sans cette tension, cette résistance, le travail du paradoxe ne pourrait avoir lieu; sans cette contrariété, l'écriture perdrait son principe, ainsi que l'explique Michon :

Il me semble qu'un écrivain (du moins en Occident depuis quelques siècles) est d'abord contemporain d'un état du monde qui l'écrase, d'un état des lettres au service de ce monde, toutes choses qu'il réprouve [...]; mais il est aussi contemporain d'un état de la langue dont il fait une arme, et grâce à quoi il transforme son refus, le fait changer de signe dans le plus haut assentiment d'une œuvre. (2007, p. 17)

Rolin, de même, définit de façon particulièrement claire cette tension :

Je fais l'hypothèse que le fait d'être «mal placé» dans son époque est plus ou moins la condition de possibilité de toute littérature, et sans doute de tout art. En d'autres termes, que la « modernité » d'une œuvre n'est pas forcément en rapport direct avec l'adhésion de l'auteur à son époque. [...] Je forme l'hypothèse que la force qui porte à écrire et soutient dans la « création continuée » de l'écriture naît de l'opposition entre un désir d'appartenance et une impossibilité d'appartenir, ou encore entre la quête d'un lieu et la fatalité d'un non-lieu. (2004, p. 24-25) 
Il ne s'agit donc pas, non plus, de renouveler le topos de l'écrivain maudit, de l'ermite. Le cœur de la démonstration est plutôt de placer au principe de l'écriture littéraire une contemporanéité mais une contemporanéité vécue comme une violence.

Nombre des obsessions, des contradictions, des distinctions qui traversent les réflexions sur la littérature des écrivains Verdier semblent ainsi difficiles à comprendre si on ne met en face d'elles les assertions sartriennes. Le discours paraît irrésistiblement attiré vers les problématiques que Sartre aura contribué à imposer dans le champ intellectuel et littéraire. La question "pour qui écrit-on?», qui fut plus largement celle de toutes les approches de la littérature soucieuses de conjuguer les progressismes esthétiques et politiques, apparaît comme une clé de voûte de la pensée de ces écrivains contemporains. Elle implique en effet la définition même de la place de l'écrivain dans la société, la qualification de son rôle. C'est notamment en raison de leur incapacité à toucher le plus grand nombre que l'on a entériné l'échec des avant-gardes à la fin des années 1970. Et de ce point précis, Sartre et Lévy n'avaient pas fini de discuter (1991). En dépit des tentatives de générations successives - et du Sartre des entretiens de 1980 lui-même - pour « décomposer » la figure sartrienne, celle-ci paraît donc hanter encore les écrivains contemporains, quitte à ce que ce soit via la référence aux plus illustres de ses contradicteurs: Barthes, Blanchot, Simon, Bourdieu ou Gracq. L'entretien contradictoire de la génération de 68 avec Sartre se poursuit ainsi souterrainement et mêle de nombreuses voix. 


\section{Bibliographie}

BERGouniouX, Pierre (1975). «L'écriture contre la littérature : Flaubert », Dialectiques, no 8, p. 69-82.

- (1991). "Le Tremblement authentique », entretien avec François Bon, Quai Voltaire, no 3, automne, p. 16-21.

- (2001). Intervention dans Politis, dossier «Littérature. L'engagement aujourd'hui », nº 642, mars, p. 35.

- (2002). «Entretien avec Pierre Bergounioux», La Licorne, « Dialogues contemporains », nº 1, p. 15-30.

Bergounioux, Gabriel et Pierre (2002). Pierre Bergounioux, l'héritage, Rencontres, Paris, Les Flohic Éditeurs, coll. « Les singuliers/Littérature ».

BobILLIER, Gérard (1992). "Verdier : treize ans de fraternité », Le Matricule des Anges, no 1, nov., $<$ http://www.lmda.net/mat/MAT00105.html>.

Bon, François (1993). "Aller arrière », Quai Voltaire, <http://www.tierslivre.net/spip/spip.php?article789>

- (1995). "Centre noir, langue cassée », entretien réalisé par Jean-Christophe Millois, Prétexte, no 7, octobre-décembre, $<\underline{\text { http://perso.club-internet.fr/pretexte/revue/entretiens/entretiens fr/entretiens/francois- }}$ bonhtm $>$

- (1998). «Sur le roman», entretien réalisé par Jean-Claude Lebrun, L'Humanité, 20 mars, <http://www.tierslivre.net/arch/itw Lebrun.html> 
- (2005). «Ensba, 10. Balzac », Cours de littérature aux BeauxArts (Paris), dans «François Bon, le blog|journal», <http://www.tierslivre.net/spip/spip.php?article76>

- (2000). Lettre à Sylviane Coyault-Dublanchet du 27 janvier (extrait), dans Sylviane Coyault-Dublanchet (2002), La Province en héritage: Pierre Michon, Pierre Bergounioux, Richard Millet, Genève, Droz, coll. "Histoire des idées et critique littéraire ».

DAENINCKX, Didier (1999 [1984]). Meurtres pour mémoire, lecture accompagnée par Marianne Genzling, Paris, La Bibliothèque Gallimard, coll. « Texte et dossier ».

ÉDITIONS VERDIER (2009). Verdier, 30 ans d'édition, catalogue général 1979-2009.

LÉVY, Benny (1984). Le Nom de l'homme : dialogue avec Sartre, Lagrasse, Verdier.

- (2005). La Cérémonie de la naissance, éd. Gilles Hanus, Lagrasse, Verdier.

- (2007). Pouvoir et liberté (cahiers de travail en dialogue avec Sartre, 1975-1980), éd. Gilles Hanus, Lagrasse, Verdier.

LÉVY, Benny et Jean-Paul SARTRE (1991). L'Espoir maintenant: les entretiens de 1980, Lagrasse, Verdier.

MichEL, Natacha (1998). L'Écrivain pensif, Lagrasse, Verdier, coll. « Philia ».

Michon, Pierre (2007). Le Roi vient quand il veut. Propos sur la littérature, Paris, Albin Michel.

RAIMOND, Michel (1989). "L'intellectuel et le gourou », Mesure, José Corti, no 2 . 
Rolin, Olivier (2004). "Un écrivain doit-il aimer son époque?», dans Anne Curien, Écrire au présent, Paris, Éditions de la Maison des sciences de l'homme, p. 24-29.

- (2008). "Comme le signe de l'infini, comme un sablier», dans Luc Rasson et Bruno Tritsmans (dir.), Olivier Rolin: Littérature, Histoire, Voyage, Amsterdam/New York, Rodopi, «CRIN », p. 147-159.

SARTRE, Jean-Paul (1972). Plaidoyer pour les intellectuels, Paris, Gallimard, coll. « Idées ».

- (1979). «L'écriture et la publication. Entretien avec Michel Sicard », Obliques, no 18-19.

Simon, Claude (1986). Discours de Stockholm, Paris, Minuit.

\section{Résumé}

Nées en quelque sorte de la rencontre de Jean-Paul Sartre et de Benny Lévy à la fin des années 1970, les éditions Verdier ont aujourd'hui réussi à fédérer autour d'elles des écrivains importants de la littérature française contemporaine. Certains de ces écrivains (Pierre Bergounioux, François Bon, Didier Daeninckx, Pierre Michon, Natacha Michel et Olivier Rolin) et les éditeurs Verdier eux-mêmes ont en commun d'être passés de l'engagement gauchiste dans les années 1970 à l'engagement dans la littérature au début des années 1980. L'article pose alors ces questions: que reste-t-il, dans le discours sur la littérature de ces auteurs, des grands concepts, des grandes idées de Sartre? Quelles fonctions sociales assignent-ils à la littérature et pour qui affirment-ils écrire? 


\begin{abstract}
Arising from the meeting of Jean-Paul Sartre and Benny Levy in the late 70s, Éditions Verdier have now attracted important writers of contemporary French literature. These writers (Pierre Bergounioux, François Bon, Didier Daeninckx, Pierre Michon, Natacha Michel and Olivier Rolin) and Verdier as publishers have in common that they went from leftist commitment in the 70 s to commitment in literature in the early 80s. The article raises the following questions: in the discourse on literature of these writers, what remains of Sartre's major concepts and main ideas? What social functions do they assign to literature? And for whom do they write?
\end{abstract}

